\title{
Cold molecular gas in the Perseus cluster core ${ }^{\star}$
}

\section{Association with X-ray cavity, $\mathrm{H} \alpha$ filaments and cooling flow}

\author{
P. Salomé ${ }^{1}$, F. Combes ${ }^{2}$, A. C. Edge ${ }^{3}$, C. Crawford ${ }^{4}$, M. Erlund ${ }^{4}$, A. C. Fabian ${ }^{4}$, N. A. Hatch ${ }^{4}$, R. M. Johnstone ${ }^{4}$, \\ J. S. Sanders ${ }^{4}$, and R. J. Wilman ${ }^{3}$
}

1 Institut de Radioastronomie Millimétrique, 300 rue de la Piscine, 38406 St. Martin d'Hères, France e-mail: salome@iram.fr

2 Observatoire de Paris, LERMA, 61 Av. de l'Observatoire, 75014 Paris, France

e-mail: francoise.combes@obspm.fr

3 Department of Physics, University of Durham, South Road, Durham DH1 3LE, UK

e-mail: alastair.edge@durham.ac.uk

4 Institute of Astronomy, Madingley Road, Cambridge CB3 OHA, UK

Received 21 December 2005 / Accepted 15 February 2006

\begin{abstract}
Cold molecular gas has recently been detected in several cooling flow clusters of galaxies containing huge optical nebula. These optical filaments are tightly linked to cooling flows and related phenomena, such as rising bubbles of relativistic plasma fed by radio jets. We present here a map, in the $\mathrm{CO}(2-1)$ rotational line, of the cold molecular gas associated with some of the $\mathrm{H} \alpha$ filaments surrounding the central galaxy of the Perseus cluster: NGC 1275. The map, extending to about $50 \mathrm{kpc}(135 \mathrm{arcsec})$ from the center of the galaxy, has been made with the 18-receiver array HERA at the focus of the IRAM $30 \mathrm{~m}$ telescope. Although most of the cold gas is concentrated to the center of the galaxy, the $\mathrm{CO}$ emission is also clearly associated with the extended filaments conspicuous in ionised gas, and could trace a possible reservoir fueling the star formation there. Some of the $\mathrm{CO}$ emission is also found where the X-ray gas could cool down more efficiently at the rims of the central X-ray cavities (where the hot gas is thought to have been pushed out and compressed by the expanding radio lobes of the central AGN). The $\mathrm{CO}$ global kinematics do not show any rotation in NGC 1275. The cold gas is probably a mixture of gas falling down on the central galaxy and of uplifted gas dragged out by a rising bubble in the intracluster medium. As recently suggested in other cluster cores, the cold gas peculiar morphology and kinematics argue for the picture of an intermittent cooling flow scenario where the central AGN plays an important role.
\end{abstract}

Key words. galaxies: cooling flows - intergalactic medium - galaxies: clusters: individual: NGC 1275 galaxies: elliptical and lenticular, $\mathrm{cD}$

\section{Introduction}

Our view on cooling flows at the core of galaxy clusters has changed considerably in recent years due to X-ray observations with Chandra and XMM-Newton (Fabian et al. 2003). This generation of X-ray satellites have found no evidence for gas below one third of the virial temperature (Allen et al. 2001; Peterson et al. 2003) and they suggested the necessity of some re-heating mechanism (Peterson et al. 2003).

At the same time, $\mathrm{CO}$ emission lines have been detected in several cooling flows at millimetre wavelengths, with the IRAM $30 \mathrm{~m}$ telescope, the James Clerk Maxwell Telescope (JCMT) and the Caltech Submillimeter Observatory (CSO) (Edge 2001; Salomé \& Combes 2003). For the first time, the presence of very cold molecular gas within these environments has been revealed. The Owens Valley Radio Observatory (OVRO) and the Plateau de Bure (PdB) interferometers have even produced the first maps of the molecular emission and confirmed the peculiar morphology and dynamics of the cold component (Edge \& Frayer 2003; Salomé \& Combes 2004a,b). The gas masses derived from these $\mathrm{CO}$ observations are also

$\star$ Table 3 is only available in electronic form at http://www . edpsciences.org consistent with the cold residual gas expected to cool out of the $\mathrm{X}$-ray band (from Chandra and XMM-Newton mass deposition rates), making it possible that the long searched for cool gas has indeed been detected.

The giant cD galaxy NGC 1275 lies in the center of the Perseus cluster (Abell 426), the X-ray brightest cluster of galaxies in the sky. This galaxy is at a redshift of 0.01756 . At this distance, $1^{\prime \prime}$ is $370 \mathrm{pc}$, with $H_{0}=70 \mathrm{~km} \mathrm{~s}^{-1} \mathrm{Mpc}^{-1}$. The gas in the core of this cluster will cool if there is no balancing heat source, due to the short cooling time of the intracluster medium (ICM) inside a few tens of kpc (Fabian et al. 2003).

The Perseus cluster (first detected in mm by Mirabel et al. 1989) remained the only cooling flow cluster core mapped in CO for approximately 10 years. During this time the origin of the molecular emission was probed by various maps that were limited in both size and sensitivity. Reuter et al. (1993) imaged the millimetric emission of the central cluster region in $\mathrm{CO}(1-$ $0)$ and $\mathrm{CO}(2-1)$ emission lines with the IRAM $30 \mathrm{~m}$ telescope and built the first map of the central 50". Braine et al. (1995) then observed the Perseus cluster in $\mathrm{CO}(1-0)$ with the IRAM Plateau de Bure interferometer (PdBI). Emission was detected around the nucleus but the continuum source made the map very noisy at the center. The authors suggested that the molecular gas could come from a source other than a cooling flow, such as 
a recent merger event. More recently, Inoue et al. (1996a) observed the cluster center with the Nobeyama Millimeter Array in $\mathrm{CO}(1-0)$ using a primary beam of $65^{\prime \prime}$ in diameter. Two peaks were identified within the inner $3 \mathrm{kpc}(8 \operatorname{arcsec})$, which may be parts of a ring-like orbiting gas structure around the nucleus that could trace the AGN fueling by the cD galaxy. Finally, the most recent millimetric map of the central 1' region has been made by Bridges \& Irwin (1998) with the JCMT in CO(2-1) and $\mathrm{CO}(3-2)$ emission lines in single dish mode. Detections out to $36^{\prime \prime}$ have been claimed, with a spatial resolution of $21^{\prime \prime}$.

Large optical nebulae are often observed within cooling flow clusters of galaxies (Crawford et al. 1999), and not detected surround galaxies in clusters where the radiative cooling time is larger than the age of the cluster. The Perseus cluster harbors a huge $\mathrm{H} \alpha$ filamentary nebula (Hu et al. 1983; McNamara et al. 1996a; Conselice et al. 2001). The origins of the optical filaments and their ionization source are not identified yet. However they do trace, in some part, the radiative cooling of the hot intracluster medium either directly or indirectly. Fabian et al. (2003) compared the X-ray structures with the optical emission and proposed that the $\mathrm{H} \alpha$ filaments could be ionized cold gas that has been drawn up behind a rising bubbles of relativistic plasma, a picture that takes into account the role of a central radio source in cooling flows (see also Boehringer et al. 1993).

The strong $\mathrm{H} \alpha$ emission in cooling flows also traces the presence of cold molecular gas. There is a strong correlation between $\mathrm{H} \alpha$ and $\mathrm{CO}$, at 10-100 K (Edge 2001; Salomé \& Combes 2003), which is reinforced by a clear association, both in terms of morphology and dynamics. This is revealed in the IRAM PdBI $\mathrm{CO}(1-0)$ and $\mathrm{CO}(2-1)$ maps of Abell 1795. $\mathrm{H} \alpha$ also correlates with the presence of warm $\mathrm{H}_{2}$, at $\sim 1000-2000 \mathrm{~K}$ (Edge et al. 2002; Wilman et al. 2002). Recent United Kingdom InfraRed Telescope (UKIRT) observations (Hatch et al. 2005a) have shown a direct association between this $\mathrm{H}_{2}$ emission and the outer optical filaments in NGC 1275. Based on near-IR Integral Field Unit (IFU) observations of the warm $\mathrm{H}_{2}$, Wilman et al. (2005) found a 50 pc radius ring in the central part of the galaxy.

To probe the link between the molecular gas and the optical filaments within cooling flows, we made a large map of the central region of NGC 1275 in $\mathrm{CO}(2-1)$ emission. The next section presents the observational procedure and the data reduction. In Sects. 3 and 4, the molecular gas emission detected with HERA on the $30 \mathrm{~m}$ telescope is presented. The implication of this new view of the Perseus cluster core through millimetric wavelength is then discussed in Sect. 5, before concluding in Sect. 6.

\section{Observations and data reduction}

The observations were made from 1st to 3rd January 2005 at the IRAM-30 m telescope. We used the HEterodyne Receiver Array HERA (Schuster et al. 2004), a focal array of 18 SIS receivers, 9 for each polarization, tuned to the $\mathrm{CO}(2-1)$ line for NGC 1275 $(226.56 \mathrm{GHz})$. The 9 pixels are arranged in the form of a center-filled square and are separated by $24^{\prime \prime}$. The sampling was 6 arcsec (full sampling), and a homogeneous mapping procedure was used to regularly sweep a $12 \times 12$ pixel map, filling the intrinsic square of $66 \times 66^{\prime \prime}$. Four such maps were created, covering the central $138 \times 138^{\prime \prime}$, with a 5 th one covering $66 \times 66^{\prime \prime}$ over the northern vertical $\mathrm{H} \alpha$ filament, centered at $(0,108)$. In total the map includes 720 points. The parameters for NGC 1275 are summarized in Table 1.

At $226 \mathrm{GHz}$, the telescope half-power beam width is $12^{\prime \prime}$. The main-beam efficiency is $\eta_{\mathrm{mb}}=T_{\mathrm{A}}^{*} / T_{\mathrm{mb}}=0.57$. The typical system temperature varied between 250 and $650 \mathrm{~K}$ (on the $T_{\mathrm{A}}^{*}$
Table 1. Parameters for NGC 1275 (at the distance of 77 Mpc adopted for NGC $1275,1 \operatorname{arcsec}=0.37 \mathrm{kpc}$ ).

\begin{tabular}{lrrrr}
\hline \hline Source & $\begin{array}{r}\text { RA } \\
(\mathrm{J} 2000.0)\end{array}$ & $\begin{array}{r}\text { Dec } \\
(\mathrm{J} 2000.0)\end{array}$ & $\begin{array}{r}V_{\text {Hel }} \\
\left(\mathrm{km} \mathrm{s}^{-1}\right)\end{array}$ & $\begin{array}{r}\text { Frequency } \\
\mathrm{GHz}\end{array}$ \\
\hline NGC 1275 & $03: 19: 48.16$ & $+41: 30: 42.1$ & +5264 & 226.560 \\
\hline
\end{tabular}

* Tuning frequency at $\mathrm{CO}(2-1)$.

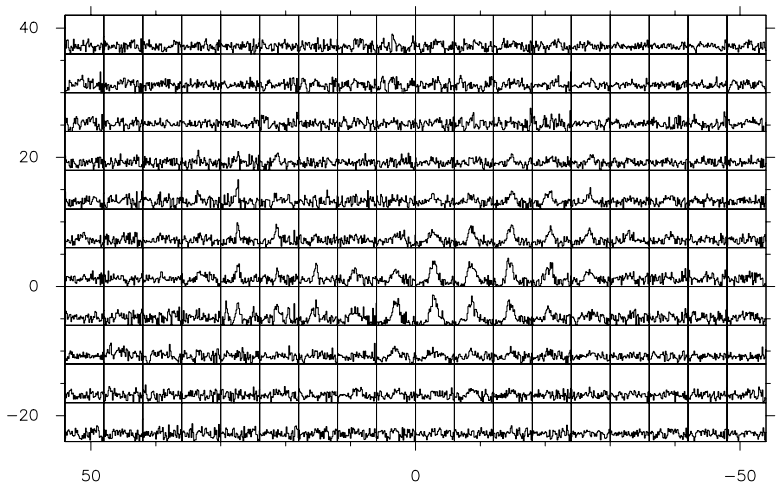

Fig. 1. Map of the central spectra taken towards NGC 1275. Each spectrum has a velocity scale from -600 to $600 \mathrm{~km} \mathrm{~s}^{-1}$, and a temperature scale in $T_{\mathrm{A}}^{*}$ from -10 to $40 \mathrm{mK}$. The positional scale shows RA-Dec in arcsec, centered on the $3 \mathrm{C} 84$ radio source.

scale). Wobbler switching mode was used, with reference positions offset by $4^{\prime}$ in azimuth. The pointing was regularly checked on NGC 1275 itself (3C 84 continuum source) and the accuracy was $3^{\prime \prime}$ rms. The WILMA backend was used, providing a band $1 \mathrm{GHz}$ wide for each of the 18 detectors. The bands contain 512 spectral channels spaced by $2 \mathrm{MHz}$. The total bandwidth corresponds to $1300 \mathrm{~km} \mathrm{~s}^{-1}$ at the $\mathrm{CO}(2-1)$ line (with velocity resolution of $2.6 \mathrm{~km} \mathrm{~s}^{-1}$ ).

The data were reduced with the GILDAS software. Misfunctioning pixels were completely rejected (3 out of 18). Some spectra with random highly non-linear baselines were suppressed. Linear baselines were subtracted from all other spectra, but the continuum at the center was impossible to detect because of its varying level (likely due to to varying atmosphere). The final spectra were smoothed by $30 \mathrm{~km} \mathrm{~s}^{-1}$.

\section{Morphology of the cold molecular gas}

\subsection{East-west emission around the central $c D$ galaxy}

The major part of the $\mathrm{CO}$ emission comes from the central region. Some of the 720 spectra (the central ones) are displayed in Fig. 1. The center of Perseus is clearly detected, and corresponds to the maximum of $\mathrm{CO}(2-1)$ emission. There is an offset with respect to the AGN center. The bulk of the molecular gas is shifted toward the West by $\sim 3 \mathrm{kpc}(8 \operatorname{arcsec})$. In addition, there is a clear detection of $\mathrm{CO}$ gas associated with the $\mathrm{H} \alpha$ emission towards the East and the West of the optical galaxy. Its total extent is around $30 \mathrm{kpc}(80 \mathrm{arcsec})$. No emission was detected in the Eastern region in CO(1-0) by Inoue et al. (1996b), probably because the emission was resolved out by their interferometer with its primary beam of 1 arcmin. The total integrated emission is plotted in Fig. 2. 

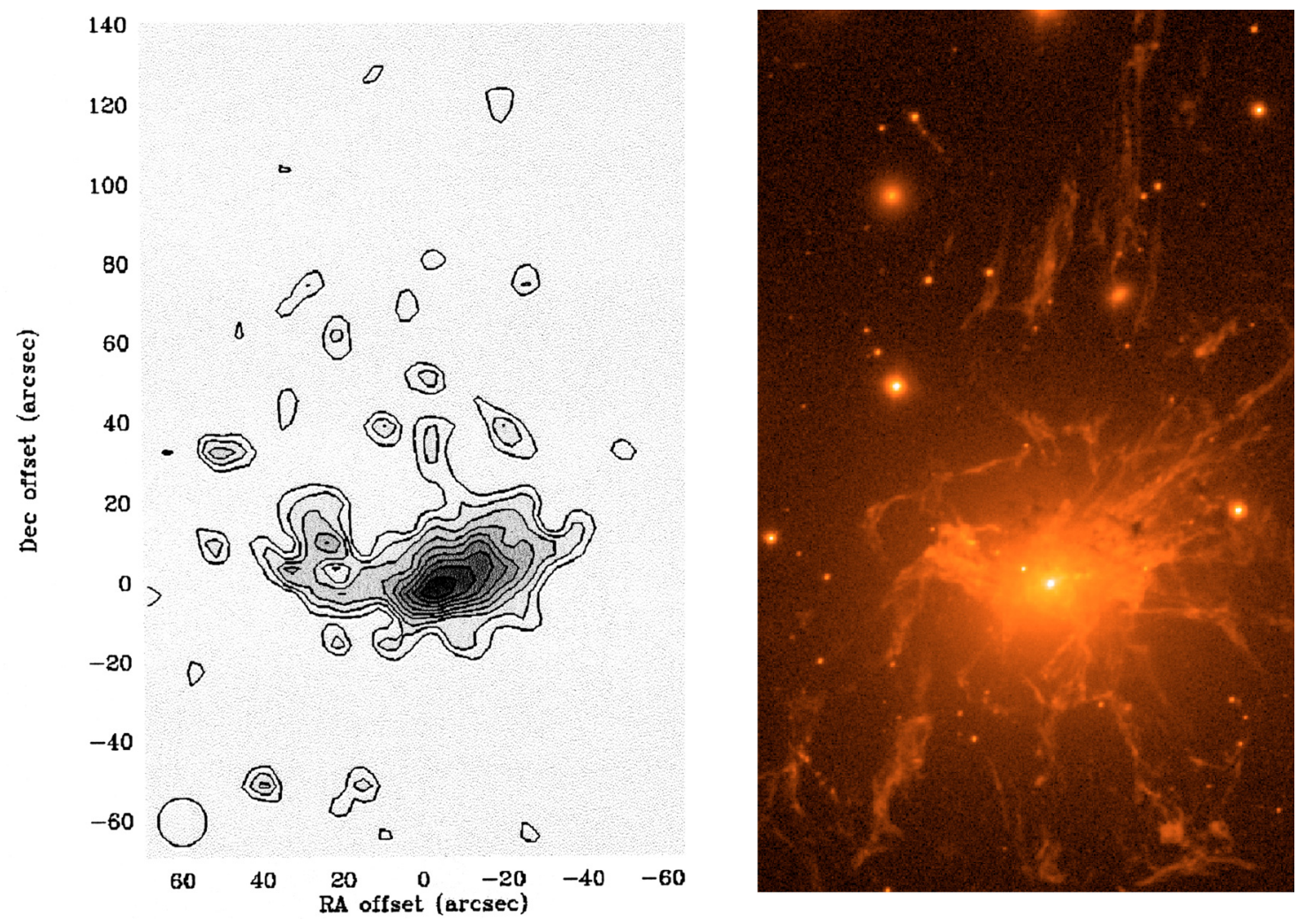

Fig. 2. Left side: integrated emission in $\mathrm{CO}(2-1)$ over the whole map. The region covered by the HERA observations is a central $138 \times 138^{\prime \prime}$ region, with a northern $66 \times 66^{\prime \prime}$ additional region centered at $\left(0,108^{\prime \prime}\right)$ to cover the vertical northern filaments. Contours are linear, from 10 to $100 \%$ of the maximum emission of $8.3 \mathrm{~K} \mathrm{~km} \mathrm{~s}^{-1}$, in $T_{\mathrm{A}}^{*}$ scale. The beam of $12^{\prime \prime}$ is indicated at the bottom left. Right side: H $\alpha$ image with the same scale (Conselice et al. 2001).

\subsection{Filamentary emission out to very large radii}

The cold molecular gas is also detected around all of the central $30 \mathrm{kpc}(80 \mathrm{arcsec})$. The emission is fainter than in the centre, and follows the filamentary $\mathrm{H} \alpha$ emission. We have extracted 115 spectra with $S / N \geq 4$, being particularly careful of broad emission lines (width $\leq 300 \mathrm{~km} \mathrm{~s}^{-1}$ ). Figure 3 shows the positions of $\mathrm{CO}(2-1)$ selected spectra compared to the regions where Conselice et al. (2001) extracted the spectra of the ionized gas. A summary of the $\mathrm{CO}$ emission line parameters is given in Tables 3 and 3 in the Appendix. The cold gas is still detectable between 20 and $50 \mathrm{kpc}(50-120$ arcsec) from the central galaxy, where the cooling time of the X-ray ICM is still low $\left(2-3 \times 10^{8} \mathrm{yr}\right.$ Sanders et al. 2004).

\subsection{Cold molecular mass distribution}

We computed the total molecular gas mass, deduced from the integrated $\mathrm{CO}$ emission, using simple assumptions about its excitation and metallicity. We assumed that the antenna temperature in $\mathrm{CO}(2-1)$ is on average 0.7 times that in the $\mathrm{CO}(1-0)$ line, over the whole surface of the emission. In the center, the $\mathrm{CO}(2-1) / \mathrm{CO}(1-0)$ ratio is equal to 1 , decreasing down to 0.5 towards the outer parts, according to Reuter et al. (1993). Bridges $\&$ Irwin (1998) measured an average ratio of 0.74 . Then we adopt the standard $\mathrm{CO}$ to $\mathrm{H}_{2}$ conversion ratio, which should apply to solar metallicity gas, of $2.3 \times 10^{20} \mathrm{~cm}^{-2}$ (Solomon et al. 1997).

We evaluated the total mass by adding the contribution of all the different regions listed in Table 3 (Appendix), taking into account overlapping beams. The total mass found is $4 \times 10^{10} M_{\odot}$, a large amount for a single galaxy. This is only a lower limit as the gas cooling from the ICM should have low metallicity. We have plotted, in Fig. 4, the local and accumulated molecular gas mass versus radius. The present results are compatible with that from Reuter et al. (1993) and Bridges \& Irwin (1998), who found a molecular gas mass close to the accumulated mass deduced here for a comparable radius. We find a large mass of gas, which is very spatially extended, showing that there is a lot of cold gas accompanying the filaments. The older measurements of Lazareff et al. (1989) and Mirabel et al. (1989) are slightly below the mass value we find with HERA.

The amount of cold gas in the core of the Perseus cluster is in agreement with the quantity of residual cooled gas expected from recent cooling rates of the intra-cluster medium. Bregman et al. (2005) deduced a mass deposition rate of $50 M_{\odot} / \mathrm{yr}$ from OVI emission, detected with FUSE from the $\sim 11 \mathrm{kpc}$ (30 arcsec) central region. X-ray data lead to $\sim 20 M_{\odot} / \mathrm{yr}$ (Fabian et al. 2005) from the same region. So the molecular gas detected here could have been accumulated in $\sim 1 \times 10^{9} \mathrm{yr}$, which is $3-4$ cooling times in that region.

\section{Dynamics of the cold molecular gas}

\subsection{Absence of rotation in the $c D$ potential well}

The kinematics deduced from the CO spectra are not regular. Relative to the systemic velocity, there are negative velocities on both sides (West and East) of the major axis of the emission, with positive velocities in the center (see the isovelocity curves in Fig. 5). 

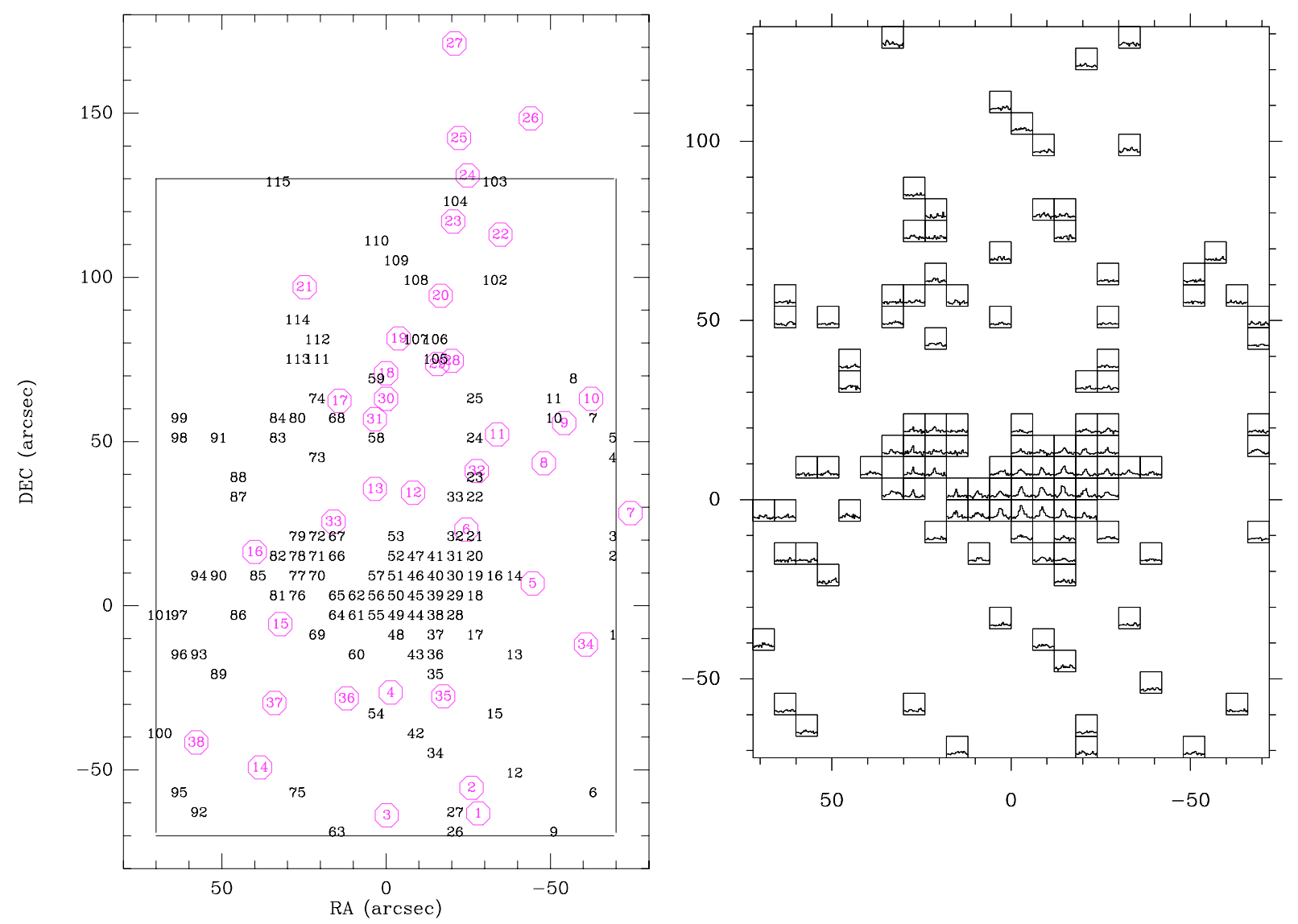

Fig. 3. Left side: positions of selected $\mathrm{CO}(2-1)$ spectra (in black) together with the $\mathrm{H} \alpha$ regions (in circles) observed by Conselice et al. (2001). Black numbers refer to regions described in Table 3. Circled red numbers refer to positions of Conselice's Fig. 5 regions. Right side: the brightest $\mathrm{CO}(2-1)$ spectra along the $\mathrm{H} \alpha$ filaments.

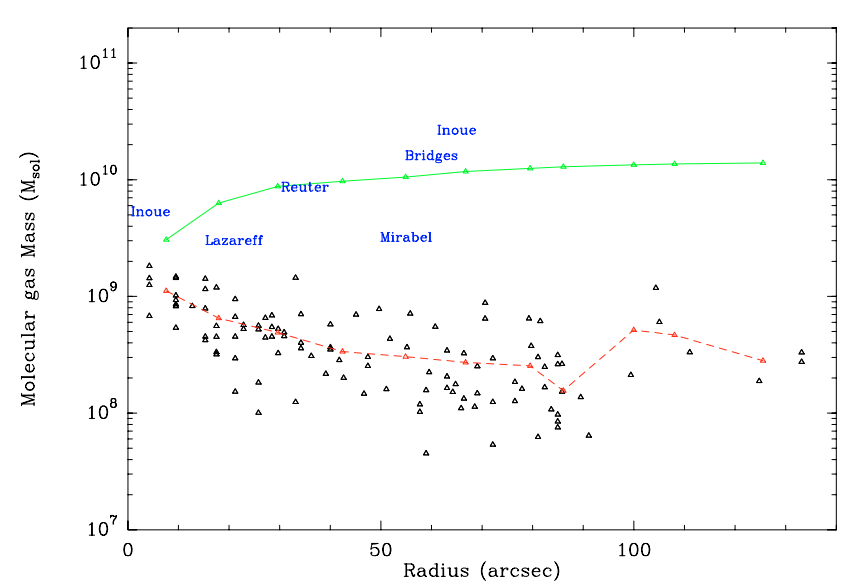

Fig. 4. Cold molecular gas mass evaluated from each fitted $\mathrm{CO}(2-1)$ line versus radius. The mean mass per point at each radius is overlaid using a dashed line. The continuous line represents the total accumulated mass at radius $\leq r$. Also shown are previous measurements with the author name.

The average of all of the selected spectra, plotted in Fig. 6, shows that the total emission cannot be fitted properly by a single Gaussian. A two component model gives better results.

We computed the CO velocity as a function of radius (Fig. 7). Each position in this diagram has been identified by a number which is referenced in Tables 3 and 3. The velocity of the $\mathrm{H} \alpha$ gas, computed by Conselice et al. (2001), has been added in red, covering the central $13 \mathrm{kpc}(35 \mathrm{arcsec})$ region with a large scatter.

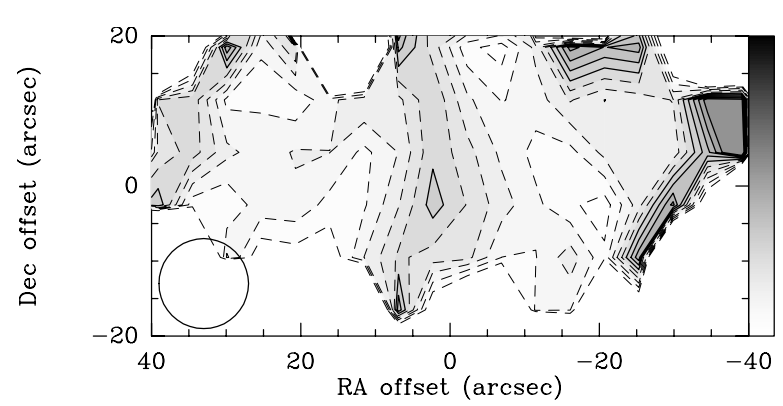

Fig. 5. Isovelocity map of the $\mathrm{CO}(2-1)$ emission. The contours are from -100 to $80 \mathrm{~km} \mathrm{~s}^{-1}$, increasing in steps of $20 \mathrm{~km} \mathrm{~s}^{-1}$. The beam of $12^{\prime \prime}$ is indicated at the bottom left. Negative velocities are shown as dashed contours.

A comparison with a typical rotation curve expected for a spherical mass model for a galaxy like NGC 1275 shows that there is no clear sign of a rotating pattern of the CO gas. Our observations show that the $\mathrm{CO}$ gas does not follow a rotational pattern, as was hinted at by previous observations (Reuter et al. 1993). The points are distributed over all the bound region. The cold gas that lies between 18.5 and $37 \mathrm{kpc}(50-100$ arcsec) is detected at a velocity of approximately $200 \mathrm{~km} \mathrm{~s}^{-1}$ in the $\mathrm{cD}$ galaxy rest frame. If the $\mathrm{CO}$ is forming from a cooling flow, we expect the gas to cool down in the cluster rest frame before being accreted by the $\mathrm{cD}$ galaxy. The cluster redshift is 0.0183 , which represents $+220 \mathrm{~km} \mathrm{~s}^{-1}$ in the cD rest frame. In Fig. 7, we have separated the points at a radius below $13 \mathrm{kpc}(35 \mathrm{arcsec})$ from the 


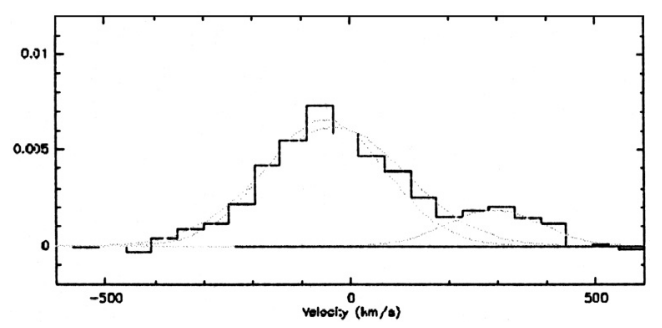

Fig. 6. Average of the selected spectra plotted in Fig. $3\left(T_{\mathrm{A}}(\mathrm{K})\right.$ vs Velocity). Overlaid are 2 Gaussian profiles with positions $292.6 \mathrm{~km} \mathrm{~s}^{-1}$ and $-51.3 \mathrm{~km} \mathrm{~s}^{-1}$, and widths $226 \mathrm{~km} \mathrm{~s}^{-1}$ and $286 \mathrm{~km} \mathrm{~s}^{-1}$.

points at a radius above this arbitrary limit, by horizontal dashed line. In the filament, the $\mathrm{CO}$ clouds velocities are between the cluster and the central $\mathrm{cD}$ velocities. However, it is not possible to discern any clear velocity gradient here.

\subsection{Two major components}

Two different trends can be identified. Close to the centre (within $35^{\prime \prime}$ ) there is an offset of $\sim-150 \mathrm{~km} \mathrm{~s}^{-1}$ between the redshift of the optical galaxy and the CO rest frame. This offset might be an indication that the gas being accreted into the potential well of the central galaxy is not yet completely relaxed. Figure 7 compares the $\mathrm{CO}(2-1)$ and $\mathrm{H} \alpha$ kinematics. The velocities of the CO line are between $0 \mathrm{~km} \mathrm{~s}^{-1}$ and $-100 \mathrm{~km} \mathrm{~s}^{-1}$, whilst the $\mathrm{H} \alpha$ velocities are spread over $\pm 250 \mathrm{~km} \mathrm{~s}^{-1}$. Nevertheless most of the $\mathrm{H} \alpha$ regions detected close the $\mathrm{CO}$ regions also have negative velocities.

At larger radii, the $\mathrm{CO}$ velocities have a larger scatter, but on average are positive. This component at large radii, which is associated with the long laminar $\mathrm{H} \alpha$ filaments may trace gas cooling out of the hot ICM that surrounds the cD galaxy. Such a reservoir of cold gas in the filaments could fuel star formation at large distances from the central galaxy, helping to photoionize the surrounding gas.

This scenario is reinforced by the velocity dispersion measured from each of the lines. Figure 8 shows the $\mathrm{CO}(2-1)$ line widths versus radius. The data inside $13 \mathrm{kpc}$ ( 35 arcsec) have a mean velocity dispersion of around $\sim 250 \mathrm{~km} \mathrm{~s}^{-1}$, whilst in the outer region this value drops to $\sim 125 \mathrm{~km} \mathrm{~s}^{-1}$ which is still quite a large value. Some of these points have an extremely high velocity dispersion. These emitters could belong to cooling filaments expected to be dynamically perturbed and kinematically dissociated from the central $\mathrm{cD}$. However, the high velocity dispersion values could also be due to the large spread of data points where the emission is fainter (in particular the outer regions).

The geometry of the source and the dynamical interaction between the ICM and the central radio lobes makes a detailed interpretation difficult. It is possible, as suggested by Fabian et al. (2001), that part of the X-ray/H $\alpha$ emission traces cooled uplifted gas, dragged behind the expanding radio lobes. An X-ray excess is also found at the edges of the radio lobes. It is likely that some of the $\mathrm{CO}$ formed in these cooler regions in the form of dense clumpy clouds. The gas probably originates from a mixture of different cooling processes occurring in a complex cooling flow scenario where the AGN plays a important role. The AGN reheats the ICM, as well as causing enhanced cooling along the radio edges, and forms bubbles of relativistic plasma which drag cooler gas from the central regions (e.g. Crawford et al. 2005b; Crawford et al. 2005a).
North, 30 kpc (80 arcsec), NGC 1275 harbors a long, thin optical filament extending radially North-South. Hatch et al. (2005b) determined the kinematics of the $\mathrm{H} \alpha$ and [NII] emission lines along this filament. The molecular gas that is detected in the same region is found to share the same velocity structure (positions $105,106,102,104,103)$. H $\alpha$ spectroscopy by Hatch et al. (2005b) revealed that the Northern and the Southern regions of the filament can be separated into two parts flowing in opposite directions. The authors suggest this implies the gas is not only falling on the central galaxy, but also flowing away along the filament. Therefore there must be a mechanism that is able to draw the gas away from the central $\mathrm{cD}$. Whether the ICM gas moves outward before it cools down to very low temperatures (10$100 \mathrm{~K}$ ) is still an open question. However, small dense clouds of molecular gas are very likely to be detected where they have formed if they are not perturbed by any external gravitational force. Whilst the central and eastern regions appear to be gas condensing and accumulating; however, it is not clear whether the extended regions represent an inflow or an outflow of cool gas. In the scenario where the radio emitting plasma from the central AGN forms buoyant bubbles rising into the ICM dragging cool gas with them, could the cold material also be dragged out, reducing the amount of mass accumulating onto the central galaxy? Comparison of $\mathrm{H} \alpha$ and $\mathrm{CO}$ kinematics along the socalled Horseshoe (a filament extended Northwest of NGC 1275) does not give a clear answer. Hatch et al. (2005b) showed that the optical filament is most likely to be flowing out behind a rising bubble, the $\mathrm{CO}$ spectra are not sensitive enough to show whether or not they follow this dynamical model (positions 4 , 10, 11 agree whilst positions 5, 7, 8 present some discrepancies). The molecular gas emission is very faint in these regions and it is hard to conclude anything from the present work. Deeper observations of these regions in the millimeter are required to accurately compare the optical and millimeter gas dynamics in the filaments.

\section{Discussion}

The $\mathrm{CO}(2-1)$ observations allow us to probe the origin of the molecular gas in NGC 1275. The chaotic kinematics are not compatible with a rotating disk, but supports the view of a system far from equilibrium. This may be due to either a recent merger, or gas accumulating from the cooling flow that has been agitated by the radio-jets. The presence of young stellar clusters in the center of NGC 1275 may be a consequence of such gas accumulation.

\subsection{Tidal interaction or cooling flow}

High resolution HST images reveal active star forming regions in the center of NGC 1275 (Holtzman et al. 1992; Carlson et al. 1998). The interpretation of the formation of these young globular clusters is debated. They may have formed during a recent merger. Alternatively, the stars may have formed out of the cooling X-ray gas, which is believed to be an intermittent phenomenon, and may be able to produce a burst of star formation. There are stellar clusters associated with the high velocity $\left(8200 \mathrm{~km} \mathrm{~s}^{-1}\right)$ system which is observed in absorption in the optical and X-ray emission. There are also stellar clusters detected as far as $22 \mathrm{kpc}(60 \mathrm{arcsec})$ from the $\mathrm{cD}$ galaxy center (Conselice et al. 2001). However, these outer stellar clusters are not preferentially associated with the $\mathrm{H} \alpha$ filaments, nor with the $\mathrm{CO}$ emission. 

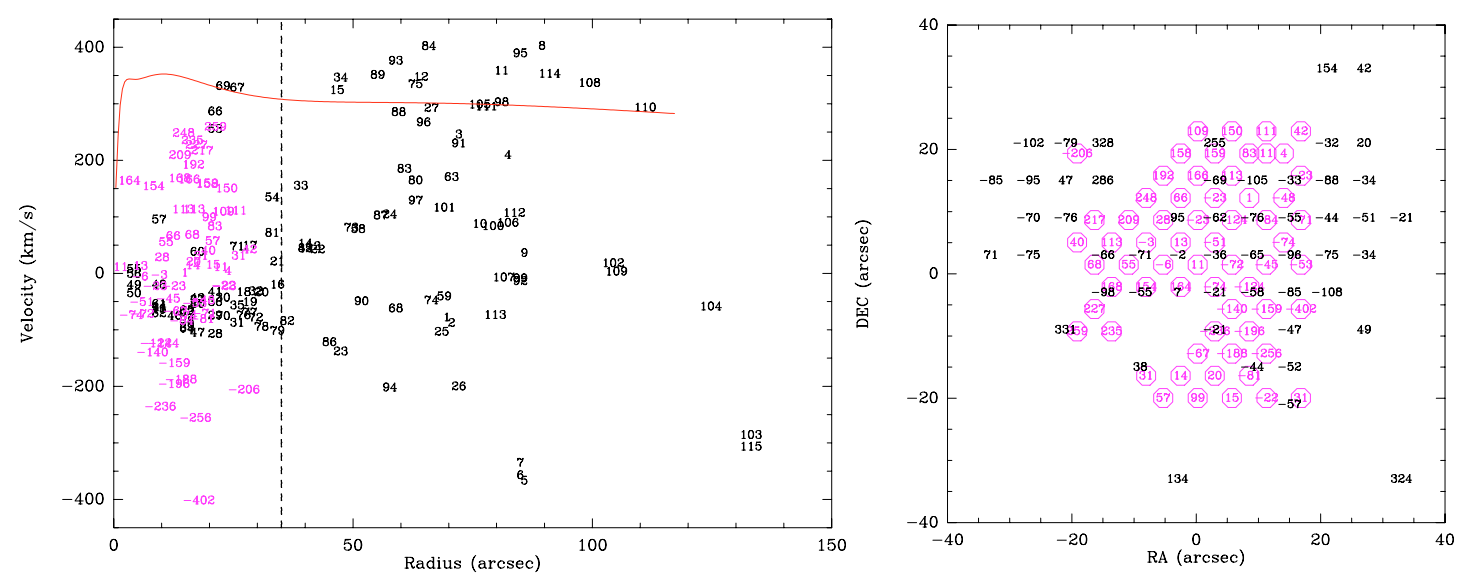

Fig. 7. Left side: $\mathrm{CO}(2-1)$ velocities versus radius plotted in black. $\mathrm{H} \alpha$ velocity distribution from Conselice et al. (2001) plotted in purple. Overlaid in red, the rotation curve expected for gas bounded in a galactic potential created by a bulge $\left(M_{\text {bulge }}=3 \times 10^{10} M_{\odot}, r_{\text {bulge }}=0.6 \mathrm{kpc}\right)$, a disk $\left(M_{\text {disk }}=1.4 \times 10^{11} M_{\odot}, r_{\text {disk }}=3.5 \mathrm{kpc}\right)$, a Black Hole $\left(M_{\mathrm{BH}}=4 \times 10^{8} M_{\odot}\right)$ and a Dark matter Halo $\left(M_{\mathrm{DM}}=5 \times 10^{11} M_{\odot}\right.$ in $r_{\mathrm{DM}}=42 \mathrm{kpc}$, with then $\left.M(r) \propto r^{2}\right)$. Right side: comparison of the $\mathrm{CO}(2-1)$ velocity with the $\mathrm{H} \alpha$ filament velocity in the central region of Perseus.

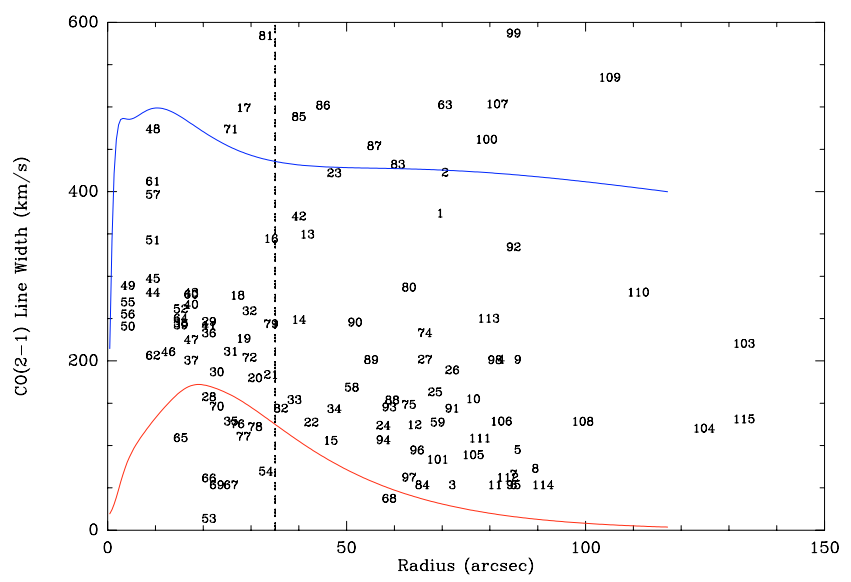

Fig. 8. $\mathrm{CO}(2-1)$ line width radial distribution. Plotted in blue is the escape velocity computed from the mass distribution shown in Fig. 7. Plotted in red is $V_{\text {crit }}=3.36 \mathrm{G} \Sigma / \kappa$, the Toomre lower limit of the velocity dispersion for a rotating gas disk with $\Sigma=500 M_{\odot} \mathrm{pc}^{-2}$ to ensure gas stability.

The merger hypothesis was proposed after early optical spectroscopy revealed two systems along the line of sight to NGC 1275 (Rubin et al. 1977): a smooth luminosity profile, early-type system, i.e. NGC 1275 itself, at $V=5200 \mathrm{~km} \mathrm{~s}^{-1}$, and superposed in front, a dusty late-type system, that obscures the low velocity system to the North. The second system emits highionisation line emission at a higher velocity of $V=8200 \mathrm{~km} \mathrm{~s}^{-1}$, but no continuum or absorption lines are detected. The system generally extends to the Northwest. The detection of a broad absorption feature in HI (van Gorkom \& Ekers 1983) without any accompanying emission does not support the hypothesis that this is a late-type system. Although the fact that no stellar component is detected may be due to obscuration, by the edge-on orientation of the disk. However, near-infrared ( $J H K$ ) maps from 2MASS detect no foreground galaxy (Jarrett et al. 2003).

In their optical study, Rubin et al. (1977) and later Unger et al. (1990) found that emission from the Northwest extension is detected at both velocities ( 5200 and $8200 \mathrm{~km} \mathrm{~s}^{-1}$ ), which supports the view that an interaction between the two systems is taking place. Moreover, gas at intermediate velocities has also been observed by Ferruit et al. (1997). It is interesting to note that the CO(1-0) map by Inoue et al. (1996b) found a Northwest extension in molecular gas that corresponds with the optical extension, at the low-velocity of $5200 \mathrm{~km} \mathrm{~s}^{-1}$. The high-velocity gas may be debris from tidal or ram pressure stripping that may interact with the gas accumulated by the cooling intracluster medium Hu et al. (1983). However, X-ray absorption data shows that the high velocity system is at least at $60 \mathrm{kpc}$ in front of NGC 1275 and therefore cannot be interacting Gillmon et al. (2004).

The eastern part of NGC 1275 appears free from the highvelocity system and so the interpretation is easier. The $\mathrm{H} \alpha$ filaments surround NGC 1275 and appear tightly correlated with the X-ray bubbles (Fabian et al. 2003). The fact that the $\mathrm{CO}(2-1)$ emission is clearly associated with the $\mathrm{H} \alpha$ filaments supports the hypothesis that the cold molecular gas radiatively cools out of the intracluster medium and that both gas components share the same excitation source.

\subsection{Excitation mechanisms of the gas}

Figure 10 compares the $\mathrm{CO}$ intensity (Ico in $\mathrm{K} \mathrm{km} \mathrm{s}^{-1}$ ) and the $\mathrm{H} \alpha$ flux ( $\mathrm{erg} \mathrm{s}^{-1} \mathrm{~cm}^{-2}$ ) radial distributions. We have normalized both emission lines by their respective maximum. The Ico decreases steeply with radius. We have superposed a curve proportional to $r^{-2}$ to mimic a central excitation source without any further losses due to attenuation. It is not clear whether we can rule out such a source of energy, since the data points are highly scattered. Conselice et al. (2001) excluded the AGN as the main ionization source. Therefore extra emission associated with star formation (for example shocks or UV radiation from young stars) in the filaments may be involved.

We compared the molecular gas mass to the $\mathrm{H} \alpha$ luminosity for the individual regions for which both lines were detected (Fig. 9). We included the measurements obtained by Edge (2001) in this plot and Salomé \& Combes (2003) for the ensemble of $\mathrm{CO}$ detected cooling flow clusters. The straight line over-plotted is the linear relation fitted by Salomé \& Combes (2003), and suggested by Edge (2001). Although NGC 1275 itself lies below the line in the Edge (2001) version of this plot, the individual regions follow the linear relation.

\subsection{Intermittent cooling flow scenario}

The CO contours appear to surround the Northern X-ray cavity which coincides with the Northern radio lobe (Fig. 11). The hot 


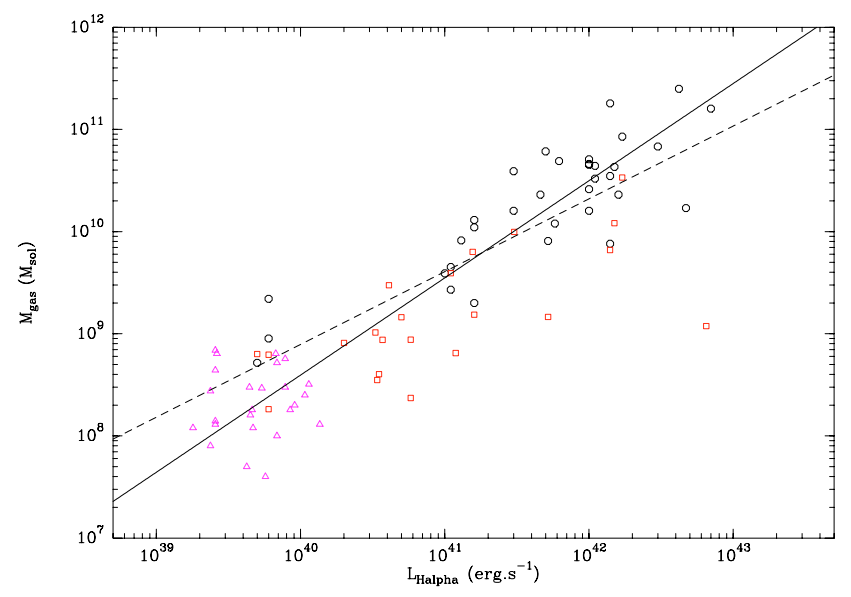

Fig. 9. Cold molecular gas mass as a function of $\mathrm{H} \alpha$ luminosity for the regions in the filaments detected at both wavelengths (see Table 3). Circles are CO detections from Edge et al. (2001), squares are CO detections from Salomé \& Combes (2004a) and triangles are present results. Overlaid in dashed line is the linear relation fitted by Salomé \& Combes (2003). Added in continuous line is a better relation which include low mass regions detected in NGC 1275 which also follow this relation.

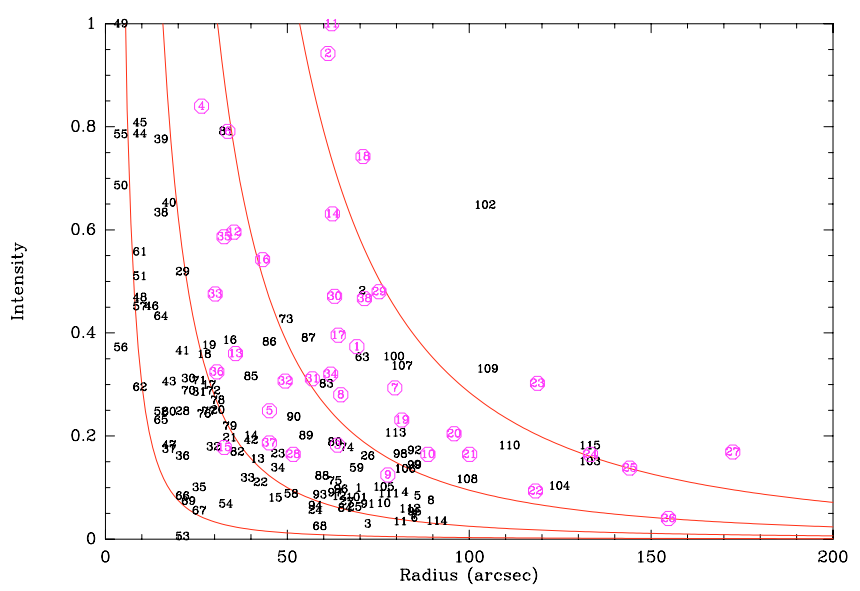

Fig. 10. Normalized $\mathrm{CO}(2-1)$ intensity (Ico in $\mathrm{K} \mathrm{km} \mathrm{s}^{-1}$ ) of selected spectra vs. radius together with normalized $\mathrm{H} \alpha\left(\mathrm{erg} \mathrm{cm}^{-2} \mathrm{~s}^{-1}\right)$ flux from Conselice et al. (2001), cf Fig. 3. Overlaid is a grid of $\propto r^{-2}$ curves.

gas, which is compressed towards the rims by the radio emitting plasma, cools more efficiently. This may explain the presence of $\mathrm{CO}$ gas in this region. This is similar to the results using the IRAM plateau de Bure interferometer, for the cooling flow cluster Abell 1795 (Salomé \& Combes 2004a). In Abell 1795 the $\mathrm{H} \alpha$ is enhanced, and $\mathrm{CO}$ is detected along the edges of the radio lobes. The alignment of $\mathrm{H} \alpha$ emission and detection of CO occurs around only one of the lobes in both NGC 1275 and Abell 1795. Active star formation is also identified in these regions. The observations presented here agree with the results from Abell 1795: the radio lobe expansion (which may re-heat the intracluster medium at large radii) can increase the radiative cooling and accelerate the formation of cold molecular clouds along the edges of the radio lobes. These cold clouds may then be accreted onto the central galaxy, or form stars along the edges of the radio lobes. A more accurate estimate of the amount of gas available for star formation compared to the amount of gas that may accrete on the $\mathrm{cD}$ galaxy is crucial to constrain the intermittent cooling flow scenario. We notice that along the edge of the Southern radio lobe, no molecular gas nor $\mathrm{H} \alpha$ emission is

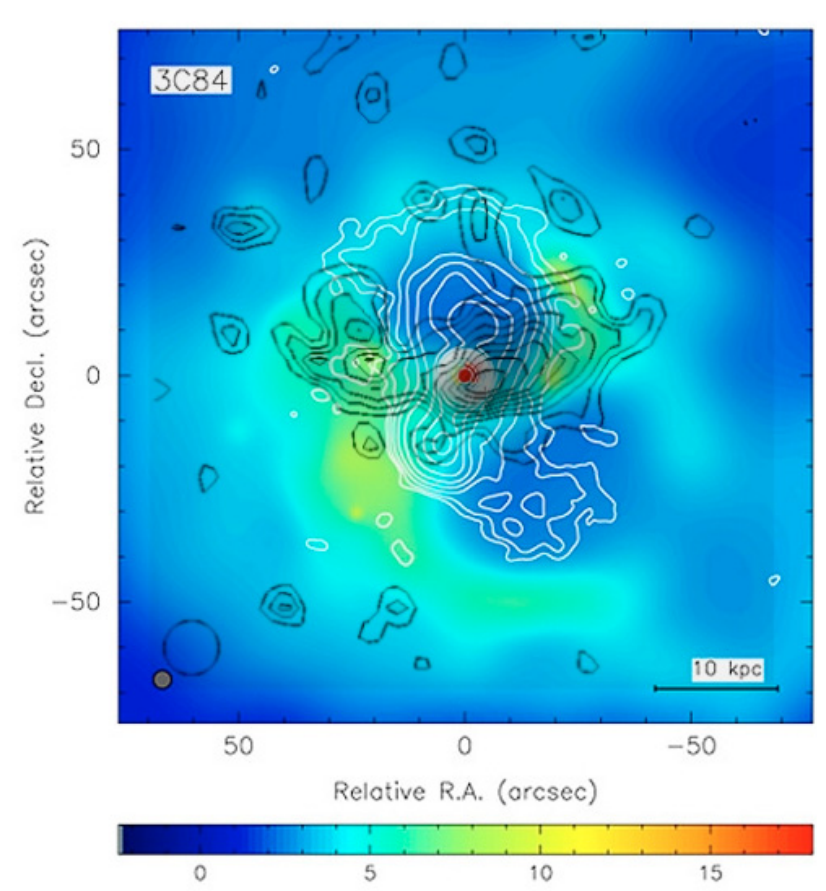

Fig. 11. Contours of the $\mathrm{CO}(2-1)$ emission (black) superimposed on the X-ray image (false colours, indicating intensity) by Fabian et al. (2003), and with the radio contours (white) by Pedlar et al. (1990). The relativistic plasma ejected by the central AGN in the two radio lobes pushes out the X-ray gas, which is compressed on the rims, and cools down there. The $\mathrm{CO}$ is also found at the border of the northern cavity.

detected, and there is no evidence of star formation. This reinforces the key role that the molecular gas plays as a fuel for star formation.

\subsection{Star formation regions inside a cooling flow}

There is a clear filamentary and clumpy extension of the $\mathrm{CO}$ emission to the East (positions 70, 71, 72, 77, 78, 79, 82, 85 ), in the same region that Hatch et al. (2005a) found $\mathrm{H}_{2}$ rovibrational lines. This region corresponds to the position of a star cluster identified by Shields \& Filippenko (1990). The warm $\mathrm{H}_{2}$ from the stellar cluster region is predominantly excited by stellar light. However, the warm molecular hydrogen detected in the outer filaments is not excited by stellar UV. Since excitation by the central AGN is insufficient, the most likely interpretation is a combination of cooling gas, thermal excitation, possibly by shocks or conduction from the intracluster medium, and an additional contribution from non-thermal excitation from stellar UV or X-rays (Hatch et al. 2005a). The cold molecular gas may be fuelling the star formation in this region. Table 2 presents the parameters of the $\mathrm{CO}$ emission lines fitted in the Shields and Filippenko star cluster region (see Fig. 12). Based on the Hatch et al. (2005a) results, we looked for two velocity components in the molecular gas lines from this region. We fitted a two component model and found a broad and narrow line Gaussian, in agreement with Hatch et al. (2005a) (line 2 and line 1 respectively in Table 2). The narrow line could be tracing the reservoir of molecular gas associated with the star forming region, while the broad line may be the underlying filament emission.

Shields \& Filippenko (1990) found a total cluster mass of $5 \times 10^{6} M_{\odot}$ (assuming that the light is dominated by O-type stars) and reached an upper limit of $7 \times 10^{7} M_{\odot}$ with a steeper IMF. This 


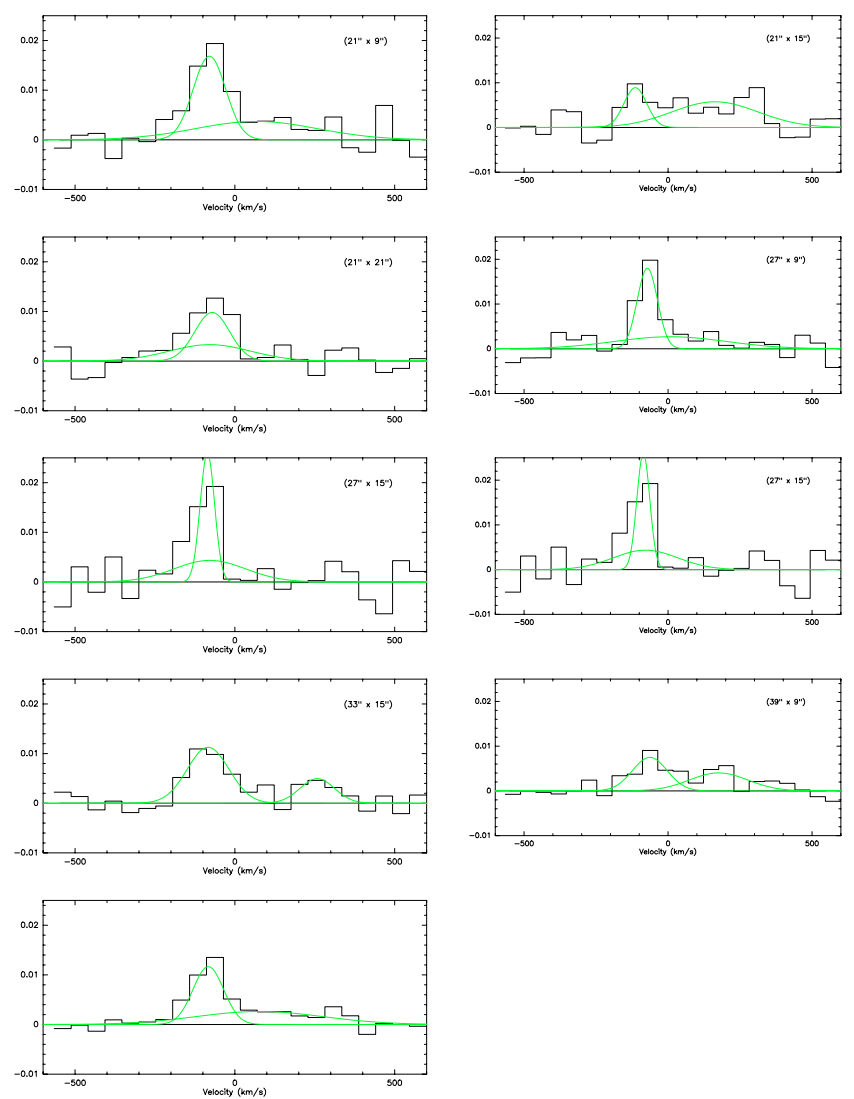

Fig. 12. Eight $\mathrm{CO}(2-1)$ spectra extracted from regions corresponding to the Shields and Filippenko star cluster region. The last spectrum at the bottom right is a line fitting the mean emission over the 8 regions displayed here. When averaging all these spectra, two distinct components can be distinguished separated by $\sim 260 \mathrm{~km} \mathrm{~s}^{-1}$, a broad line and a narrow line which could be coming from the filament and the star cluster region respectively.

is small compared to the $6 \times 10^{9} M_{\odot}$ of molecular gas found in that region, even with a very low star formation efficiency.

McNamara et al. (1996b) used optical observations to deduce a star formation rate inside the central $\sim 15 \mathrm{kpc}$ (40 arcsec) of approximately $40 M_{\odot} / \mathrm{yr}$ over $7 \times 10^{7} \mathrm{yr}$. This star formation rate is similar to the $\mathrm{X}$-ray derived mass deposition rate in the same region (20-50 $\left.M_{\odot} / \mathrm{yr}\right)$. So the radiative cooling of the hot intracluster medium may create a reservoir of cold gas. If the star formation rate is slightly smaller than the mass deposition rate $\left(\sim 5 M_{\odot} / \mathrm{yr}\right)$, then it is possible to accumulate a total mass of $2-5 \times 10^{10} M_{\odot}$ in $10 \mathrm{Gyr}$ (as deduced from the present $\mathrm{CO}$ observations in the same region). It is also possible that the cooling intracluster medium only causes intermittent star formation events while the radiative cooling of the intracluster medium is continuous.

\section{Conclusions}

The extended $\mathrm{CO}(2-1)$ map created with the HERA array at the IRAM-30 m reveals cold molecular gas sharing the same morphology as the $\mathrm{H} \alpha$ emitting gas as detected by Conselice et al. (2001). In particular both gas components share an EastWest extension. In addition strong $\mathrm{H}_{2}$ emission is detected in the Eastern filaments (Hatch et al. 2005a). The CO contours surround the Northern X-ray cavity formed by the ejection of relativistic plasma from the central AGN into the intracluster
Table 2. Intensity of the $\mathrm{CO}(2-1)$ emission lines in the Shields and Filippenko star cluster region. Two Gaussians have been fitted. Around half of the total area is found in a broad line $\left(300 \mathrm{~km} \mathrm{~s}^{-1}\right)$ and the other half is found in a narrower $\left(130 \mathrm{~km} \mathrm{~s}^{-1}\right)$ component. The stronger emission could be associated with the star cluster and the smaller one with the underlying filament.

\begin{tabular}{cccc}
\hline \hline $\begin{array}{c}\text { Position } \\
(“ \times ”)\end{array}$ & $\begin{array}{c}\text { Area 1 } \\
\left(\mathrm{K} \mathrm{km} \mathrm{s}^{-1}\right)\end{array}$ & $\begin{array}{c}\text { Area 2 } \\
\left(\mathrm{K} \mathrm{km} \mathrm{s}^{-1}\right)\end{array}$ & Area2 / (Area1+Area2) \\
\hline$(21,9)$ & $2.14 \pm 0.8$ & $1.76 \pm 1.07$ & 0.45 \\
$(21,15)$ & $0.88 \pm 0.36$ & $2.14 \pm 0.54$ & 0.7 \\
$(21,21)$ & $1.33 \pm 1.01$ & $1.05 \pm 1.07$ & 0.44 \\
$(27,9)$ & $1.58 \pm 0.39$ & $1.3 \pm 0.73$ & 0.45 \\
$(27,15)$ & $1.46 \pm 0.51$ & $1.21 \pm 0.73$ & 0.45 \\
$(27,21)$ & $0.54 \pm 0.26$ & $1.33 \pm 0.59$ & 0.7 \\
$(33,15)$ & $1.84 \pm 0.25$ & $0.66 \pm 0.22$ & 0.26 \\
$(39,9)$ & $1.13 \pm 0.18$ & $1.02 \pm 0.18$ & 0.47 \\
\hline All & $1.4 \pm 0.22$ & $1.3 \pm 0.31$ & 0.48 \\
\hline
\end{tabular}

medium. The CO kinematics do not show any rotational structure. The picture emerging from these observations is consistent with the interpretation that the hot intracluster gas has been pushed and compressed by the expanding radio lobes. Along the edges of the lobes, the gas is denser, therefore it cools more efficiently and can cool quickly to low temperatures. Molecular gas may form in these regions and be detected as $\mathrm{CO}$ emission surrounding the radio lobes. This molecular gas may fuel star formation which in turn can provide some of the photons that ionise the $\mathrm{H} \alpha$ emitting gas.

Acknowledgements. Based on observations carried out with the IRAM $30 \mathrm{~m}$ telescope. IRAM is supported by INSU/CNRS (France), MPG (Germany) and IGN (Spain). The authors also would like to acknowledge in particular the IRAM staff for help provided during the observations.

\section{References}

Allen, S. W., Fabian, A. C., Johnstone, R. M., Arnaud, K. A., \& Nulsen, P. E. J. 2001, MNRAS, 322, 589

Boehringer, H., Voges, W., Fabian, A. C., Edge, A. C., \& Neumann, D. M. 1993, MNRAS, 264, L25

Braine, J., Wyrowski, F., Radford, S. J. E., Henkel, C., \& Lesch, H. 1995, A\&A, 293, 315

Bregman, J. N., Miller, E. D., Athey, A. E., \& Irwin, J. A. 2005, ArXiv Astrophysics e-prints

Bridges, T. J., \& Irwin, J. A. 1998, MNRAS, 300, 967

Carlson, M. N., Holtzman, J. A., Watson, A. M., et al. 1998, AJ, 115, 1778

Conselice, C. J., Gallagher, J. S., \& Wyse, R. F. G. 2001, AJ, 122, 2281

Crawford, C. S., Allen, S. W., Ebeling, H., Edge, A. C., \& Fabian, A. C. 1999, MNRAS, 306, 857

Crawford, C. S., Hatch, N. A., Fabian, A. C., \& Sanders, J. S. 2005a, MNRAS, 363,216

Crawford, C. S., Sanders, J. S., \& Fabian, A. C. 2005b, MNRAS, 361, 17

Edge, A. C. 2001, MNRAS, 328, 762

Edge, A. C., \& Frayer, D. T. 2003, ApJ, 594, L13

Edge, A. C., Wilman, R. J., Johnstone, R. M., et al. 2002, MNRAS, 337, 49

Fabian, A. C., Sanders, J. S., Allen, S. W., et al. 2003, MNRAS, 344, L43

Fabian, A. C., Sanders, J. S., Ettori, S., et al. 2001, MNRAS, 321, L33

Fabian, A. C., Sanders, J. S., Taylor, G. B., et al. 2005, ArXiv Astrophysics e-prints

Ferruit, P., Adam, G., Binette, L., \& Pecontal, E. 1997, New Astron., 2, 345

Gillmon, K., Sanders, J. S., \& Fabian, A. C. 2004, MNRAS, 348, 159

Hatch, N. A., Crawford, C. S., Fabian, A. C., \& Johnstone, R. M. 2005a, MNRAS, 358, 765

Hatch, N. A., Crawford, C. S., Fabian, A. C., \& Johnstone, R. M. 2005b, ArXiv Astrophysics e-prints

Holtzman, J. A., Faber, S. M., Shaya, E. J., et al. 1992, AJ, 103, 691

Hu, E. M., Cowie, L. L., Kaaret, P., et al. 1983, ApJ, 275, L27

Inoue, M. Y., Kameno, S., Kawabe, R., et al. 1996a, AJ, 111, 1852

Inoue, M. Y., Kameno, S., Kawabe, R., et al. 1996b, AJ, 111, 1852 
Jarrett, T. H., Chester, T., Cutri, R., Schneider, S. E., \& Huchra, J. P. 2003, AJ, 125,525

Lazareff, B., Castets, A., Kim, D.-W., \& Jura, M. 1989, ApJ, 336, L13

McNamara, B. R., Jannuzi, B. T., Elston, R., Sarazin, C. L., \& Wise, M. 1996a, ApJ, 469, 66

McNamara, B. R., O'Connell, R. W., \& Sarazin, C. L. 1996b, AJ, 112, 91

Mirabel, I. F., Sanders, D. B., \& Kazes, I. 1989, ApJ, 340, L9

Pedlar, A., Ghataure, H. S., Davies, R. D., et al. 1990, MNRAS, 246, 477

Peterson, J. R., Kahn, S. M., Paerels, F. B. S., et al. 2003, ApJ, 590, 207

Reuter, H. P., Pohl, M., Lesch, H., \& Sievers, A. W. 1993, A\&A, 277, 21

Rubin, V. C., Ford, W. K., Peterson, C. J., \& Oort, J. H. 1977, ApJ, 211, 693

Salomé, P., \& Combes, F. 2003, A\&A, 412, 657

Salomé, P., \& Combes, F. 2004a, A\&A, 415, L1
Salomé, P., \& Combes, F. 2004b, in SF2A-2004: Semaine de l'Astrophysique Française, meeting held in Paris, France, June 14-18, 2004, ed. F. Combes,

D. Barret, T. Contini, F. Meynadier, \& L. Pagani (EDP-Sciences), SF2A, 131 Sanders, J. S., Fabian, A. C., Allen, S. W., \& Schmidt, R. W. 2004, MNRAS, 349,952

Schuster, K.-F., Boucher, C., Brunswig, W., et al. 2004, A\&A, 423, 1171

Shields, J. C., \& Filippenko, A. V. 1990, ApJ, 353, L7

Solomon, P. M., Rivolo, A. R., Barrett, J., \& Yahil, A. 1997, ApJ, 319, 730

Unger, S. W., Taylor, K., Pedlar, A., et al. 1990, MNRAS, 242, P33

van Gorkom, J., \& Ekers, D. J. 1983, ApJ, 267, 528

Wilman, R. J., Edge, A. C., \& Johnstone, R. M. 2005, MNRAS, 359, 755

Wilman, R. J., Edge, A. C., Johnstone, R. M., et al. 2002, MNRAS, 337, 63 


\section{Online Material}


P. Salomé et al.: Cold molecular gas in the Perseus cluster core, Online Material p 2

Table 3. Parameters of the brigthest $\mathrm{CO}(2-1)$ emission lines plotted in Fig. 3. The first and second columns give the region number as used in the related plots and the corresponding position offset in arcsec relative to the $\mathrm{cD}$ coordinates. The 3rd, 4th, 5th, 6th, 7th columns list the line parameters of fitted Gaussian profiles. The center of the lines is taken by comparison with the cD rest frame $(z=0.01756)$. In Col. 8, we computed the cold molecular mass for each region, as explain in the text. Finally, the last column list the $\mathrm{H} \alpha$ flux in $10^{-15} \mathrm{erg} \mathrm{s}^{-1} \mathrm{~cm}^{-2}$ found by Conselice et al. (2001) in the region identified in the Fig. 3.

\begin{tabular}{|c|c|c|c|c|c|c|c|c|}
\hline Number & $\begin{array}{c}\text { Position } \\
\text { (“×”) }\end{array}$ & $\begin{array}{l}\text { Peak } \\
(\mathrm{mK}) \\
\end{array}$ & $S / N$ & $\begin{array}{c}\text { Area } \\
\left(\mathrm{K} \mathrm{km} \mathrm{s}^{-1}\right) \\
\end{array}$ & $\begin{array}{c}\text { Center } \\
\left(\mathrm{km} \mathrm{s}^{-1}\right) \\
\end{array}$ & $\begin{array}{c}\text { Width } \\
\left(\mathrm{km} \mathrm{s}^{-1}\right)\end{array}$ & $\begin{array}{c}M_{\mathrm{gas}} \\
\left(10^{8} M_{\odot}\right) \\
\end{array}$ & $\begin{array}{c}F(\mathrm{H} \alpha) \\
10^{15} \mathrm{erg} \mathrm{s}^{-1} \mathrm{~cm}^{-2}\end{array}$ \\
\hline 1 & $(-70,-9)$ & $2.4 \pm 0.4$ & 5.9 & $0.9 \pm 0.2$ & $-78.0 \pm 58.0$ & $374.0 \pm 63.4$ & 1.8 & 7.2 \\
\hline 2 & $(-70,15)$ & $10.5 \pm 1.3$ & 7.5 & $4.7 \pm 0.4$ & $-88.1 \pm 20.1$ & $422.1 \pm 42.3$ & 8.7 & \\
\hline 3 & $(-70,21)$ & $5.1 \pm 1.0$ & 4.7 & $0.2 \pm 0.2$ & $245.6 \pm 21.6$ & $52.9 \pm 59.7$ & 0.5 & 6.6 \\
\hline 4 & $(-70,45)$ & $4.1 \pm 0.4$ & 9.1 & $0.8 \pm 0.2$ & $209.2 \pm 21.2$ & $201.3 \pm 39.1$ & 1.6 & \\
\hline 5 & $(-70,51)$ & $8.1 \pm 1.8$ & 4.4 & $0.8 \pm 0.3$ & $-367.0 \pm 18.1$ & $94.5 \pm 45.1$ & 1.5 & \\
\hline 6 & $(-63,-58)$ & $7.1 \pm 1.6$ & 4.3 & $0.4 \pm 0.1$ & $-357.3 \pm 7.2$ & $52.9 \pm 132.0$ & 0.7 & \\
\hline 7 & $(-63,57)$ & $6.5 \pm 0.9$ & 6.9 & $0.4 \pm 0.2$ & $-335.4 \pm 22.0$ & $65.6 \pm 40.4$ & 0.8 & 3.7 \\
\hline 8 & $(-58,69)$ & $9.5 \pm 0.9$ & 10.0 & $0.7 \pm 0.2$ & $402.5 \pm 8.4$ & $72.3 \pm 20.4$ & 1.3 & \\
\hline 9 & $(-52,-70)$ & $6.6 \pm 1.1$ & 5.8 & $1.4 \pm 0.3$ & $35.7 \pm 21.5$ & $201.1 \pm 48.7$ & 2.6 & \\
\hline 10 & $(-52,57)$ & $4.1 \pm 0.8$ & 5.0 & $0.6 \pm 0.2$ & $87.4 \pm 28.6$ & $154.6 \pm 55.6$ & 1.2 & 2.8 \\
\hline 11 & $(-52,63)$ & $5.9 \pm 0.9$ & 6.5 & $0.3 \pm 0.1$ & $358.6 \pm 24.0$ & $52.9 \pm 81.6$ & 0.6 & \\
\hline 12 & $(-39,-52)$ & $6.1 \pm 0.9$ & 6.2 & $0.8 \pm 0.3$ & $347.4 \pm 20.2$ & $123.9 \pm 38.9$ & 1.5 & \\
\hline 13 & $(-39,-16)$ & $4.1 \pm 1.0$ & 4.0 & $1.5 \pm 0.2$ & $47.9 \pm 23.8$ & $349.1 \pm 75.2$ & 2.8 & \\
\hline 14 & $(-39,9)$ & $7.4 \pm 1.0$ & 7.2 & $1.9 \pm 0.3$ & $52.7 \pm 16.4$ & $248.3 \pm 35.2$ & 3.6 & \\
\hline 15 & $(-33,-33)$ & $7 \pm 1.6$ & 4.3 & $0.7 \pm 0.2$ & $324.0 \pm 11.5$ & $105.4 \pm 21.8$ & 1.4 & \\
\hline 16 & $(-33,9)$ & $10.3 \pm 1.3$ & 7.5 & $3.7 \pm 0.5$ & $-20.0 \pm 20.7$ & $343.9 \pm 74.4$ & 7.0 & \\
\hline 17 & $(-28,-9)$ & $5.5 \pm 1.0$ & 5.4 & $2.9 \pm 0.3$ & $49.1 \pm 30.0$ & $498.4 \pm 70.5$ & 5.4 & \\
\hline 18 & $(-28,3)$ & $11.9 \pm 1.3$ & 8.7 & $3.5 \pm 0.3$ & $-33.3 \pm 15.3$ & $276.9 \pm 32.4$ & 6.5 & \\
\hline 19 & $(-28,9)$ & $15.3 \pm 1.4$ & 10.7 & $3.6 \pm 0.3$ & $-50.9 \pm 11.0$ & $225.9 \pm 28.1$ & 6.8 & \\
\hline 20 & $(-28,15)$ & $12.8 \pm 0.9$ & 13.8 & $2.4 \pm 0.2$ & $-33.8 \pm 7.9$ & $179.3 \pm 20.4$ & 4.5 & \\
\hline 21 & $(-28,21)$ & $9.9 \pm 0.6$ & 16.1 & $1.9 \pm 0.3$ & $20.6 \pm 16.8$ & $183.1 \pm 40.1$ & 3.5 & 17.8 \\
\hline 22 & $(-28,33)$ & $7.9 \pm 0.9$ & 8.8 & $1.0 \pm 0.2$ & $42 \pm 16.0$ & $126.8 \pm 37.2$ & 2 & \\
\hline 23 & $(-28,39)$ & $3.6 \pm 0.8$ & 4.3 & $1.6 \pm 0.2$ & $-138.4 \pm 31.3$ & $421.4 \pm 70.5$ & 3.0 & 6.9 \\
\hline 24 & $(-28,51)$ & $4.1 \pm 0.8$ & 5.0 & $0.5 \pm 0.2$ & $104.1 \pm 18.5$ & $123.2 \pm 35.8$ & 1.0 & \\
\hline 25 & $(-28,63)$ & $3.5 \pm 0.8$ & 4.0 & $0.6 \pm 0.2$ & $-103.5 \pm 30.2$ & $162.6 \pm 44.1$ & 1.1 & \\
\hline 26 & $(-22,-70)$ & $7.8 \pm 1.5$ & 4.9 & $1.5 \pm 0.5$ & $-200.2 \pm 33.7$ & $188.6 \pm 93.5$ & 2.94 & 8.4 \\
\hline 27 & $(-22,-63)$ & $3.3 \pm 0.4$ & 6.9 & $0.7 \pm 0.3$ & $292.4 \pm 45.3$ & $201.2 \pm 136.5$ & 1.3 & 21.2 \\
\hline 28 & $(-22,-4)$ & $14.5 \pm 3.3$ & 4.3 & $2.4 \pm 0.4$ & $-107.3 \pm 12.3$ & $156.9 \pm 29.9$ & 4.5 & \\
\hline 29 & $(-22,3)$ & $19.4 \pm 2.9$ & 6.6 & $5.0 \pm 0.4$ & $-74.7 \pm 10.0$ & $245.9 \pm 21$ & 9.4 & \\
\hline 30 & $(-22,9)$ & $15.4 \pm 1.6$ & 9.1 & $3.0 \pm 0.3$ & $-43.1 \pm 9.2$ & $186.4 \pm 20.0$ & 5.6 & \\
\hline 31 & $(-22,15)$ & $12.4 \pm 1$ & 12.3 & $2.7 \pm 0.3$ & $-87.4 \pm 13.6$ & $210.6 \pm 24.8$ & 5.2 & \\
\hline 32 & $(-22,21)$ & $6.3 \pm 0.7$ & 8.0 & $1.7 \pm 0.3$ & $-31.8 \pm 22.4$ & $258.4 \pm 45.7$ & 3.2 & 17.8 \\
\hline 33 & $(-22,33)$ & $7.1 \pm 1.0$ & 6.5 & $1.1 \pm 0.3$ & $154.9 \pm 22.0$ & $153.5 \pm 52.8$ & 2.1 & \\
\hline 34 & $(-16,-46)$ & $8.9 \pm 1.4$ & 6.0 & $1.3 \pm 0.5$ & $346.3 \pm 24.4$ & $142.8 \pm 63.7$ & 2.5 & \\
\hline 35 & $(-16,-22)$ & $7.2 \pm 1.3$ & 5.4 & $0.9 \pm 0.4$ & $-56.9 \pm 26.6$ & $128.1 \pm 76.1$ & 1.8 & 13.2 \\
\hline 36 & $(-16,-16)$ & $6.3 \pm 1.1$ & 5.6 & $1.5 \pm 0.2$ & $-51.0 \pm 18.7$ & $232.3 \pm 35.8$ & 2.9 & \\
\hline 37 & $(-16,-9)$ & $8.0 \pm 1.6$ & 4.7 & $1.7 \pm 0.4$ & $-46.5 \pm 22.5$ & $200.1 \pm 52.7$ & 3.1 & \\
\hline 38 & $(-16,-4)$ & $23.9 \pm 1.3$ & 17.7 & $6.1 \pm 0.4$ & $-84.3 \pm 7.5$ & $244.1 \pm 17.8$ & 11.56 & \\
\hline 39 & $(-16,3)$ & $29.5 \pm 2.1$ & 13.7 & $7.5 \pm 0.4$ & $-95.4 \pm 6.2$ & $241.8 \pm 14.5$ & 14.1 & \\
\hline 40 & $(-16,9)$ & $22.6 \pm 1.4$ & 15.3 & $6.3 \pm 0.5$ & $-54.0 \pm 10.4$ & $266.1 \pm 29.2$ & 11.9 & \\
\hline 41 & $(-16,15)$ & $13.9 \pm 1.7$ & 7.8 & $3.5 \pm 0.4$ & $-32.9 \pm 12.0$ & $241.2 \pm 30.8$ & 6.6 & \\
\hline 42 & $(-9,-39)$ & $4.7 \pm 0.7$ & 6.2 & $1.8 \pm 0.3$ & $44.0 \pm 37.0$ & $370.3 \pm 61.2$ & 3.5 & \\
\hline 43 & $(-10,-16)$ & $10.0 \pm 2.3$ & 4.3 & $2.9 \pm 0.5$ & $-43.8 \pm 25.4$ & $280.3 \pm 53.6$ & 5.5 & \\
\hline 44 & $(-9,-4)$ & $25.8 \pm 1.4$ & 18.2 & $7.6 \pm 0.4$ & $-57.8 \pm 7.6$ & $280.5 \pm 21.7$ & 14.3 & \\
\hline 45 & $(-10,3)$ & $25.1 \pm 1.9$ & 13.1 & $7.9 \pm 0.5$ & $-64.0 \pm 9.8$ & $296.8 \pm 22.3$ & 14.7 & \\
\hline 46 & $(-10,9)$ & $19.8 \pm 0.6$ & 31.5 & $4.4 \pm 0.2$ & $-75.1 \pm 5.8$ & $210.2 \pm 13.1$ & 8.2 & \\
\hline 47 & $(-10,15)$ & $7.4 \pm 1.2$ & 6.0 & $1.7 \pm 0.4$ & $-104.9 \pm 27.1$ & $224.5 \pm 64.6$ & 3.3 & \\
\hline 48 & $(-4,-9)$ & $9.1 \pm 1.8$ & 4.9 & $4.5 \pm 0.4$ & $-20.0 \pm 21.0$ & $473.5 \pm 43.5$ & 8.5 & \\
\hline 49 & $(-4,-4)$ & $31.9 \pm 1.4$ & 21.5 & $9.7 \pm 0.3$ & $-20.6 \pm 4.4$ & $288.5 \pm 11.7$ & 18.2 & \\
\hline 50 & $(-4,3)$ & $26.3 \pm 1.4$ & 17.6 & $6.7 \pm 0.2$ & $-35.3 \pm 4.2$ & $240.3 \pm 9.9$ & 12.5 & \\
\hline 51 & $(-4,9)$ & $13.7 \pm 1.4$ & 9.5 & $4.9 \pm 0.3$ & $-61.4 \pm 10.0$ & $342.2 \pm 23.2$ & 9.3 & \\
\hline 52 & $(-4,15)$ & $8.7 \pm 1.5$ & 5.7 & $2.4 \pm 0.3$ & $-68.0 \pm 17.4$ & $260.8 \pm 48.5$ & 4.5 & \\
\hline 53 & $(-4,21)$ & $3.4 \pm 0.8$ & 4.0 & $0.1 \pm 0.2$ & $255.3 \pm 20.92$ & $13.2 \pm 29.8$ & 0.1 & \\
\hline 54 & $(3,-33)$ & $9.1 \pm 1.4$ & 6.2 & $0.6 \pm 0.2$ & $134.2 \pm 10.8$ & $69.0 \pm 26.9$ & 1.2 & 7.3 \\
\hline 55 & $(3,-4)$ & $26.9 \pm 2.1$ & 12.2 & $7.6 \pm 0.3$ & $7.3 \pm 5.7$ & $268.8 \pm 15.2$ & 14.3 & \\
\hline 56 & $(3,3)$ & $13.4 \pm 2.2$ & 6 & $3.6 \pm 0.4$ & $-1.1 \pm 14.7$ & $254.7 \pm 33.8$ & 6.7 & \\
\hline 57 & $(3,9)$ & $10.5 \pm 1.8$ & 5.5 & $4.4 \pm 0.4$ & $95.0 \pm 17.9$ & $396.0 \pm 39.0$ & 8.2 & \\
\hline 58 & $(3,51)$ & $4.8 \pm 0.8$ & 5.6 & $0.8 \pm 0.2$ & $78 \pm 18.8$ & $168.2 \pm 48.1$ & 1.6 & 7.0 \\
\hline 59 & $(3,69)$ & $10.0 \pm 2.1$ & 4.6 & $1.3 \pm 0.3$ & $-40.9 \pm 14.8$ & $126.9 \pm 29.5$ & 2.51 & 16.7 \\
\hline 60 & $(9,-16)$ & $8.1 \pm 0.7$ & 10.8 & $2.4 \pm 0.1$ & $38.0 \pm 8.6$ & $278.1 \pm 16.4$ & 4.5 & \\
\hline
\end{tabular}


P. Salomé et al.: Cold molecular gas in the Perseus cluster core, Online Material $p 3$

Table 3. continued.

\begin{tabular}{|c|c|c|c|c|c|c|c|c|}
\hline Number & $\begin{array}{l}\text { Position } \\
\text { ("X") }\end{array}$ & $\begin{array}{l}\text { Peak } \\
(\mathrm{mK})\end{array}$ & $S / N$ & $\begin{array}{c}\text { Area } \\
\left(\mathrm{K} \mathrm{km} \mathrm{s}^{-1}\right)\end{array}$ & $\begin{array}{c}\text { Center } \\
\left(\mathrm{km} \mathrm{s}^{-1}\right)\end{array}$ & $\begin{array}{c}\text { Width } \\
\left(\mathrm{km} \mathrm{s}^{-1}\right)\end{array}$ & $\begin{array}{c}M_{\mathrm{gas}} \\
\left(10^{8} M_{\odot}\right)\end{array}$ & $\begin{array}{c}F(\mathrm{H} \alpha) \\
10^{-15} \mathrm{erg} \mathrm{s}^{-1} \mathrm{~cm}^{-2}\end{array}$ \\
\hline 61 & $(9,-4)$ & $12.4 \pm 1.7$ & 7.0 & $5.4 \pm 0.3$ & $-54.0 \pm 16.3$ & $411.6 \pm 37$ & 10.1 & \\
\hline 62 & $(9,3)$ & $13.1 \pm 2.9$ & 4.4 & $2.8 \pm 0.5$ & $-70.8 \pm 21.1$ & $205.9 \pm 48.2$ & 5.3 & \\
\hline 63 & $(15,-70)$ & $6.4 \pm 1.2$ & 5.0 & $3.4 \pm 0.4$ & $170.3 \pm 31.5$ & $501.9 \pm 66.1$ & 6.4 & 4.1 \\
\hline 64 & $(15,-4)$ & $15.9 \pm 1.9$ & 7.9 & $4.2 \pm 0.5$ & $-97.8 \pm 14.6$ & $250.0 \pm 34.2$ & 7.8 & \\
\hline 65 & $(15,3)$ & $19.6 \pm 2.5$ & 7.7 & $2.2 \pm 0.2$ & $-65.6 \pm 5.4$ & $108.5 \pm 11.4$ & 4.2 & \\
\hline 66 & $(15,15)$ & $12.6 \pm 1.3$ & 9.3 & $0.8 \pm 0.2$ & $286.4 \pm 16.7$ & $61 \pm 179.0$ & 1.5 & \\
\hline 67 & $(15,21)$ & $9.6 \pm 1.9$ & 4.9 & $0.5 \pm 0.2$ & $328.4 \pm 7.5$ & $52.9 \pm 761.6$ & 1 & 10.7 \\
\hline 68 & $(15,57)$ & $6.1 \pm 1.4$ & 4.6 & $0.2 \pm 0.3$ & $-62.2 \pm 19.0$ & $37.0 \pm 31.4$ & 0.4 & 8.9 \\
\hline 69 & $(21,-9)$ & $12.8 \pm 2.3$ & 5.3 & $0.7 \pm 0.2$ & $331.2 \pm 4.6$ & $52.9 \pm 179.6$ & 1.3 & 4.0 \\
\hline 70 & $(21,9)$ & $18.2 \pm 3.2$ & 5.6 & $2.8 \pm 0.4$ & $-75.7 \pm 9.8$ & $145.8 \pm 28.9$ & 5.2 & \\
\hline 71 & $(21,15)$ & $5.9 \pm 1.4$ & 4.1 & $3 \pm 0.6$ & $47.4 \pm 40.8$ & $473.2 \pm 87.4$ & 5.6 & \\
\hline 72 & $(21,21)$ & $13.0 \pm 2.2$ & 5.7 & $2.8 \pm 0.5$ & $-78.6 \pm 14.1$ & $203.5 \pm 46.9$ & 5.2 & 10.7 \\
\hline 73 & $(21,45)$ & $5.5 \pm 1.2$ & 4.6 & $4.1 \pm 0.8$ & $80.7 \pm 16.1$ & $704.6 \pm 135.3$ & 7.7 & \\
\hline 74 & $(21,63)$ & $7.0 \pm 1.4$ & 4.7 & $1.7 \pm 0.3$ & $-48 \pm 22.0$ & $232.5 \pm 37.5$ & 3.2 & \\
\hline 75 & $(27,-58)$ & $7.0 \pm 1.1$ & 5.9 & $1.1 \pm 0.2$ & $334.5 \pm 17.0$ & $147.8 \pm 32.1$ & 2.0 & 14.2 \\
\hline 76 & $(27,3)$ & $17.9 \pm 1.5$ & 11.2 & $2.3 \pm 0.3$ & $-74.3 \pm 7.2$ & $124.9 \pm 16.4$ & 4.4 & 4.0 \\
\hline 77 & $(27,9)$ & $20.7 \pm 2.2$ & 9.1 & $2.4 \pm 0.4$ & $-69.1 \pm 7.6$ & $110.3 \pm 21.7$ & 4.5 & \\
\hline 78 & $(27,15)$ & $20.4 \pm 3.9$ & 5.1 & $2.6 \pm 0.4$ & $-94.6 \pm 10.1$ & $121.6 \pm 24.8$ & 4.9 & \\
\hline 79 & $(27,21)$ & $8.3 \pm 1.1$ & 7.0 & $2.1 \pm 0.5$ & $-101.8 \pm 25.2$ & $243.1 \pm 75.0$ & 4 & \\
\hline 80 & $(27,57)$ & $6.0 \pm 1.2$ & 4.8 & $1.8 \pm 0.4$ & $164.3 \pm 29.2$ & $286.4 \pm 61.9$ & 3.4 & \\
\hline 81 & $(33,3)$ & $12.5 \pm 2.8$ & 4.4 & $7.7 \pm 0.7$ & $71.4 \pm 25.0$ & $583.6 \pm 54.1$ & 14.4 & 4.0 \\
\hline 82 & $(33,15)$ & $10.8 \pm 1.6$ & 6.7 & $1.6 \pm 0.3$ & $-84.6 \pm 12.9$ & $143.4 \pm 28.8$ & 3.0 & 12.2 \\
\hline 83 & $(33,51)$ & $6.4 \pm 1.4$ & 4.5 & $2.9 \pm 0.2$ & $184.8 \pm 11.5$ & $431.6 \pm 26.8$ & 5.5 & \\
\hline 84 & $(33,57)$ & $10.4 \pm 1.9$ & 5.5 & $0.5 \pm 0.2$ & $401.7 \pm 9.6$ & $52.9 \pm 187.6$ & 1.0 & \\
\hline 85 & $(39,9)$ & $5.9 \pm 0.7$ & 8.0 & $3.0 \pm 0.5$ & $44.1 \pm 36.2$ & $487.9 \pm 81.6$ & 5.7 & 12.2 \\
\hline 86 & $(45,-4)$ & $7.0 \pm 1.4$ & 4.2 & $3.7 \pm 1.0$ & $-121.9 \pm 61.7$ & $501.4 \pm 201.3$ & 6.9 & 4.0 \\
\hline 87 & $(45,33)$ & $7.9 \pm 1.3$ & 5.7 & $3.8 \pm 0.3$ & $102.2 \pm 16.8$ & $453.5 \pm 33.0$ & 7.1 & \\
\hline 88 & $(45,39)$ & $7.3 \pm 1.1$ & 6.4 & $1.1 \pm 0.3$ & $285.0 \pm 14.2$ & $153.1 \pm 47.2$ & 2.2 & \\
\hline 89 & $(51,-22)$ & $9.2 \pm 1.3$ & 6.5 & $1.9 \pm 0.4$ & $351.0 \pm 22.7$ & $200.8 \pm 46.6$ & 3.6 & \\
\hline 90 & $(51,9)$ & $8.9 \pm 1.2$ & 6.8 & $2.3 \pm 0.3$ & $-49.5 \pm 14.9$ & $245.1 \pm 38.6$ & 4.3 & \\
\hline 91 & $(51,51)$ & $4.3 \pm 0.5$ & 7.4 & $0.6 \pm 0.2$ & $229.8 \pm 17.1$ & $143.4 \pm 31.8$ & 1.2 & \\
\hline 92 & $(57,-63)$ & $4.7 \pm 0.6$ & 7.3 & $1.6 \pm 0.5$ & $-13.9 \pm 64.7$ & $334 \pm 128.5$ & 3.1 & \\
\hline 93 & $(57,-16)$ & $5.6 \pm 0.8$ & 6.1 & $0.8 \pm 0.3$ & $376.0 \pm 23.7$ & $144.9 \pm 44.7$ & 1.5 & \\
\hline 94 & $(57,9)$ & $5.6 \pm 1.2$ & 4.4 & $0.6 \pm 0.2$ & $-202.0 \pm 19.4$ & $106.1 \pm 39.5$ & 1.1 & \\
\hline 95 & $(63,-58)$ & $9.2 \pm 1.2$ & 7.6 & $0.5 \pm 0.1$ & $389.1 \pm 5.2$ & $52.9 \pm 583.8$ & 0.9 & \\
\hline 96 & $(63,-16)$ & $9.4 \pm 1.7$ & 5.4 & $0.9 \pm 0.2$ & $267.0 \pm 10.4$ & $94.0 \pm 18.5$ & 1.7 & \\
\hline 97 & $(63,-4)$ & $13.3 \pm 1.3$ & 10.0 & $0.8 \pm 0.2$ & $128.7 \pm 8.2$ & $61.9 \pm 55.4$ & 1.6 & \\
\hline 98 & $(63,51)$ & $7.5 \pm 1.2$ & 5.9 & $1.6 \pm 0.3$ & $302.6 \pm 20.8$ & $200.7 \pm 38.8$ & 3.0 & \\
\hline 99 & $(63,57)$ & $2.2 \pm 0.4$ & 5.0 & $1.4 \pm 0.2$ & $-8.9 \pm 37.4$ & $586.6 \pm 83.1$ & 2.6 & \\
\hline 100 & $(69,-39)$ & $7.0 \pm 1.3$ & 5.2 & $3.4 \pm 0.5$ & $83.3 \pm 29.4$ & $461.1 \pm 73.4$ & 6.4 & 10.5 \\
\hline 101 & $(69,-4)$ & $8.9 \pm 1.3$ & 6.3 & $0.7 \pm 0.3$ & $116.2 \pm 15.3$ & $83.1 \pm 41.0$ & 1.4 & \\
\hline 102 & $(-33,99)$ & $8.6 \pm 1.1$ & 7.1 & $6.3 \pm 0.9$ & $17.8 \pm 46.9$ & $693.9 \pm 99.6$ & 11.8 & \\
\hline 103 & $(-33,129$ & $6.3 \pm 1.4$ & 4.2 & $1.4 \pm 0.5$ & $-286.1 \pm 38.2$ & $220.0 \pm 79.4$ & 2.75 & 3.7 \\
\hline 104 & $(-21,123$ & $7.9 \pm 1.3$ & 5.8 & $1 \pm 0.2$ & $-58.7 \pm 14.0$ & $119.8 \pm 35.2$ & 1.8 & \\
\hline 105 & $(-15,75)$ & $10.5 \pm 0.9$ & 10.7 & $0.9 \pm 0.3$ & $298.6 \pm 15.4$ & $88.4 \pm 25.3$ & 1.8 & \\
\hline 106 & $(-15,81)$ & $9.8 \pm 1.9$ & 4.9 & $1.3 \pm 0.3$ & $89.2 \pm 16.4$ & $128.1 \pm 37.5$ & 2.4 & \\
\hline 107 & $(-10,81)$ & $6.1 \pm 0.3$ & 15.9 & $3.2 \pm 0.5$ & $-7.4 \pm 45.1$ & $502.9 \pm 93.3$ & 6.1 & \\
\hline 108 & $(-10,99)$ & $8.3 \pm 0.6$ & 12.6 & $1.1 \pm 0.23$ & $336.7 \pm 14.2$ & $127.9 \pm 29.1$ & 2.1 & \\
\hline 109 & $(-3,105)$ & $5.6 \pm 1.0$ & 5.6 & $3.2 \pm 0.5$ & $3.0 \pm 43.1$ & $534.4 \pm 100.4$ & 6.02 & \\
\hline 110 & $(3,111)$ & $5.9 \pm 1.4$ & 4.2 & $1.7 \pm 0.4$ & $293.2 \pm 28.2$ & $280.6 \pm 55.6$ & 3.3 & \\
\hline 111 & $(20,75)$ & $7.5 \pm 1.6$ & 4.6 & $0.8 \pm 0.3$ & $295.0 \pm 26.6$ & $108.2 \pm 59.7$ & 1.6 & \\
\hline 112 & $(20,81)$ & $8.7 \pm 1.2$ & 7.0 & $0.5 \pm 0.4$ & $106.6 \pm 33.1$ & $61.7 \pm 97.8$ & 1.0 & \\
\hline 113 & $(26,75)$ & $7.61 \pm 1.3$ & 5.8 & $2.0 \pm 0.4$ & $-73.7 \pm 23.5$ & $249.6 \pm 42.8$ & 3.7 & \\
\hline 114 & $(26,87)$ & $6.1 \pm 1.4$ & 4.3 & $0.3 \pm 0.1$ & $353.2 \pm 19.0$ & $52.9 \pm 130.1$ & 0.6 & \\
\hline 115 & $(33,129)$ & $12.7 \pm 2.8$ & 4.4 & $1.7 \pm 0.4$ & $-306.6 \pm 14.9$ & $130.7 \pm 30.0$ & 3.3 & \\
\hline
\end{tabular}

